\title{
DISCUSSION (Khokhlova)
}

STEPIEN: For the work you do, it is very important to use correct model atmospheres. If one does not have a model of a particular Ap star one is studying, the question arises: which model approximation best fits an Ap star? Dr. Muthsam (Vienna) and I have produced a grid of models with different chemical compositions to compare with Osawa's star (HD 221568). When you look at $T(\tau)$ and the energy distribution in the ultraviolet and visible, you can see [shows some slides] that the model which best approximates HD 221568 is the model with solar composition increased by a factor of 30 . This means that we should use a grid of models with abundances increased by a factor of this order to use it for different Ap stars. Even with this, there are differences between our best fit and the observed flux distribution for HD 221568, but it is much closer than the model with solar composition.

KHOKHLOVA: Certainly, to use the correct model would be very nice. But I did not use Kurucz models, I used the older Mihalas (1965) models to determine the parameter $\mathrm{R}(0)_{\infty}$. Until one knows the distribution of elements, one can not use a proper blanketed model atmosphere. Remember that in our analysis we determine not the local abundance value directly, but local equivalent widths. These widths should be analysed with the aid of a proper model. There would be some sort of iteration process: as a first approximation we could get local abundances with the simplest model, then compute a new model and improve the abundance.

YAGOLA: I would like to supplement Dr. Khokhlova's report. The problem of mapping chemical elements on the surfaces of Ap stars is an example of an inverse problem in astrophysics. An astrophysicist often has to solve such problems when interpreting observational data. Unfortunately, many of these problems are incorrectly posed, so that a small change in experimental data can produce a large change in the solution. Since 1967, we have been using a stable method, a regularizing algorithm devised by Academician A. N. Tikhonov, and his scheme for solving inverse problems in astrophysics. To describe this algorithm now would be impossible, but I would like to recommend to you our books on this problem, published by Nauka Press. These are: Tikhonov, Goncharsky, Stepanov, and Yagola, A Regularizing Algorithm and A Priori Information, and two other books, The Numerical Method for Solution of Inverse Problems in Astrophysics (1978) and a new one, Incorrectly Posed Problems in Astrophysics, which should be published in September or October of this year.

MICHAUD: Have you tried to reproduce the profiles using rings, and if so, can you show how much worse the fits would be if you used rings? In other words, can you exclude rings?

KHOKHLOVA: We can not exclude rings, because in our method we can only get a map of the strip $\pm 45^{\circ}$ around the subsolar line.

MÉCESSIER: With your program, could you check whether rings can fit the observed profiles?

KHOKHLOVA: We do not fit any particular model. We solve for the distribution by inversion. The profiles are the data, and the maps are the result. [long pause] I can check any map you like!

POLOSUKHINA: How many lines were used to make the maps? Also, how 133

C. R. Cowley et al (eds.), Upper Main Sequence Stars with Anomalous Abundances, 133-134.

(C) 1986 by D. Reidel Publishing Company. 
different were the maps from a number of different lines?

KHOKHLOVA: For $\theta$ Aurigae, for example, we used eight lines of iron. All maps obtained from lines of the same element are similar.

WEISS: Several years ago, it was a commonly expressed idea that elements are concentrated either at the magnetic equator or at the magnetic poles. From your maps, I gain the impression that the spots are more or less distributed at random on the surface, and not really correlated with the magnetic field. This might cause problems for diffusion. Can you comment?

KHOKHLOVA: For $\alpha^{2} \mathrm{CVn}, \mathrm{Eu}$ is concentrated near the pole, and Cr near the equator, but $\mathrm{Fe}$ and $\mathrm{Ti}$ are more complicated. This is especially the case for $\theta$ Aur, which has a well determined rather weak magnetic field with a sinusoidal shape of the effective field variation, but a rather complicated chemical structure. Other stars, such as $\varepsilon$ UMa, $x$ Ser and CU Vir also have prominent inhomogeneities but rather weak fields.

DWORETSKY: Dr. Khokhlova, is there some reason why you have not used magnetic null lines for element mapping? This would remove the complications due to the magnetic field. Is there any possibility that you may use them in further work?

KHOKHIOVA: Yes. There are not very many magnetic null lines, and many of them are blended. The first requirement for our analysis is the absence of blending. Certainly, we shall try to find such a line in the future.

HENSBERGE: You mentioned that the deviation from simple dipole magnetic structure found apparently in some stars might be caused by the spotted distribution of elements. The strongest indication for the deviation from simple dipole geometry is given by direct measurement of the surface magnetic field $B_{S}$ in 53 Cam and HD 126515. In both stars, $B_{S}$ varies approximately from 10 to $17 \mathrm{kG}$.

I guess that it would be very hard to explain the variation of a factor 1.7 by spots and a dipole structure; for a dipole, the field strength difference between equator and poles is only a factor of two. KHOKHLOVA: For $\alpha^{2}$ CVn the centered dipole is the case, but for other stars we do not know, but might guess, that taking into account chenical inhomogenieties may change the result of measurements of magnetic fields. 